\title{
Magnetic Resonance of Microstrip Line Loaded with Split Ring Resonator and Spiral Resonator
}

\author{
Rajni', Gurwinder Singh ${ }^{2 *}$ and Anupma Marwaha ${ }^{3}$ \\ 'Department of ECE, Shaheed Bhagat Singh State Technical Campus, Ferozepur - 152004, Punjab, India; \\ rajni_c123@yahoo.co.in \\ ${ }^{2}$ Departmentof ECE, Government Polytechnic College, Ferozepur - 152002, Punjab, India; \\ gurwinder08singh@gmail.com \\ ${ }^{3}$ Department of ECE, Sant Longowal Institute of Engineering and Technology, \\ Longowal (Sangrur) - 148106, Punjab, India
}

\begin{abstract}
Background/Objectives: In this paper, magnetic resonance of a microstrip line loaded with planar square split ring resonator (SRR) and spiral resonator (SR) structure, printed on Rogers R03010 substrate, has been investigated. Methods/ Statistical analysis: The electromagnetic interaction of resonators with microstrip line is examined by simulating microstrip line loaded resonators inside a waveguide with High Frequency Structure Simulator(HFSS software). Findings: The magnetic resonance in SRR/SR loaded microstrip line shifts to lower frequency region due to loading. Further it is observed that resonant frequency shifts to lower frequency region to a great extent with SR loading as compared to SRR loading. Application/Improvements: The resonant frequency in SR loaded microstrip line is much less than resonant frequency of SRR loaded microstrip line. Hence SR loading can be used to design electrically small antennas.
\end{abstract}

Keywords: High Frequency Structure Simulator (HFSS), Magnetic resonance, Metamaterials (MTM), Microstrip Line, Split Ring Resonator (SRR), Spiral Resonator (SR)

\section{Introduction}

The rise of metamaterials has unlocked the door for designers to create structure with unusual properties or better performance ${ }^{1,2}$. Metamaterials are artificially invented materials that exhibit properties not detected in naturally occurring materials. One of the most electrifying opportunities for metamaterials is the development of negative-index metamaterials (NIMs). The first and one of the most important contributions to this topic was made in 1968 by VG Veselago who said that materials with both negative permittivity and permeability is theoretically possible ${ }^{3}$. In 1999, John Pendry identified a practical way to make Left-Handed Metamaterials (LHM) which did not follow the conventional right hand rule ${ }^{4}$. Later then, Smith and his colleagues demonstrated metamaterials to show negative permittivity and permeability simultaneously and performed microwave experiments to test its unusual features in 2000. In 2001, Smith et al. showed negative refraction experimentally, using a metamaterials with repeated unit cells of SRR and copper strips ${ }^{5-7}$. In 2002, Marques et al. in ${ }^{8}$ investigated bianisotropic behaviour of the SRR unit cell structure with broadside coupled split-ring resonators (BC-SRR) and presented comparative analysis of the conventional (or edge-coupled) SRR and BC-SRR. The BC-SRR has printed metallic rings on both sides of the dielectric substrate which are aligned in such a way that their splits are displaced by 180 degrees. Bilotti et al. in 2007 analyzed SRR unit cells with multiple rings and Spiral Resonators (SR) with multiple numbers of turn in ${ }^{9}$. They derived mathematical expressions for calculation of resonant frequency from their equivalent circuits and measured the

${ }^{*}$ Author for correspondence 
miniaturized rate and quality factor for varying number of turns in SRs and rings in multiple split ring resonators (MSRRs). The authors did parametric analysis of SR to observe the dependence of resonant frequency on geometry of resonators in $\frac{10}{10}$. They designed new left hand metmaterial unit cell of SR and Capacitive Loaded Strips

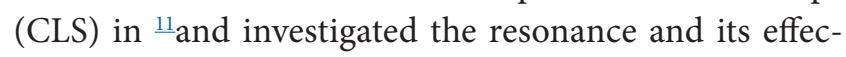
tive material parameters. In $\frac{12}{2}$, Garg et al. implemented micro-splits in SRR by means of RF MEMS switches for MTM loaded electrically small planar patch antenna. The frequency tunability is achieved with introduction of micro-splits by altering the reactance of structure. Rajaraman et al. in ${ }^{13}$ loaded basic patch with two pairs of Complementary Split Ring Resonators (CSRR's) on both sides of its non-radiating edges with different dimensions on both sides to improve gain in UMTS and ISM applications.

Baena et al. in ${ }^{14}$ reported a different approach for the development of planar metamaterial structures with Split-Ring Resonators (SRRs) and Complementary Split-Ring Resonators (CSRRs) coupled to planar transmission lines and analyzed the stop band/pass band characteristics of the SRR/CSRR loaded transmission lines. Bojanic et al. in 2011 proposed multiband delay line with broadside coupled and single split-ring resonators and exhibited two left-handed bands which could be altered by rotating the split rings for certain angle or by varying their lengths $\frac{15}{}$. In 2012, Sindreu et al. at suggested the use of SRR in coupled transmission line as compared to microwave components to achieve better performance parameters ${ }^{16}$. Naqui et al. in 2013reported the electromagnetic properties of transmission lines loaded with SRRs and CSRRs aligned in a non-orthogonal direction to the line axis ${ }^{17}$. In 2014, Bojanic et al. presented an enhanced equivalent circuit approach for the electromagnetic interaction of single SRR with printed lines and extract the different parameters of microstrip line with parallel and perpendicular gap to line $\mathrm{e}^{\frac{18}{8}}$. In $\frac{19}{}$ authors numerically analyzed electric and magnetic interaction of Double Split Ring Resonator (DSRR) and single and two-mirrored split ring resonators in different orientations (i.e. with gap of SRR parallel and perpendicular to the line).

In this paper, a microstrip line is loaded with double SRR and dual turn SR in the same plane. The loaded strip line is analyzed to compare the electromagnetic behavior of microstrip line with gap of resonators parallel and perpendicular to the microstrip line. This paper is planned in four sections. Section 1 gives a brief summary of the previous work done. Section 2 presents SRR/SR loaded microstrip line model. Results have been presented and discussed in Section 3. Conclusion of the work done is presented in Section 4.

\section{Proposed SRR/SR Loaded Microstrip Line Model}

In the proposed model, a conventional microstrip line is loaded with planar square SRR/SR in two configurations. The resonators are made up of copper metal. The SRR/SR is coupled to microstrip line by placing it at distance's', in the same plane. This coupled line is modeled on Rogers RO3010 substrate of thickness, has $1.27 \mathrm{~mm}$, dielectric permittivity, $\varepsilon_{\mathrm{r}}=10.2$ and $\tan \delta=0.035$.

The following two configurations of loaded microstrip line are considered in this work:

- SRR loaded microstrip line with the gap parallel and perpendicular to the line

- SR loaded microstrip line with the gap parallel and perpendicular to the line.

\subsection{SRR Loaded Microstrip Line with Gap Parallel/Perpendicular to The Line}

The top view of SRR coupled to microstrip line in the same plane with gap parallel and perpendicular to the line is shown in Figure 1(a) and 1(b) respectively. The various

Table1. Dimensions of microstrip line loaded SRR and SR

\begin{tabular}{|c|c|c|c|}
\hline \multicolumn{4}{|c|}{ Parameters } \\
\hline Sr. No. & $\begin{array}{c}\text { Name of } \\
\text { parameter }\end{array}$ & Representation & $\begin{array}{c}\text { Dimensions } \\
\text { (in mm })\end{array}$ \\
\hline 1 & $\begin{array}{c}\text { Width of } \\
\text { microstrip line }\end{array}$ & Wd & 1.2 \\
\hline 2 & $\begin{array}{c}\text { Length of outer } \\
\text { ring of SRR/outer } \\
\text { turn of SR }\end{array}$ & L & 3.0 \\
\hline 3 & Width of SRR/SR & W & 0.2 \\
\hline 5 & $\begin{array}{c}\text { Gap/split of } \\
\text { resonator }\end{array}$ & G & 0.5 \\
\hline & $\begin{array}{c}\text { Spacing between } \\
\text { microstrip line } \\
\text { and SRR/SR }\end{array}$ & T & 0.1 \\
\hline
\end{tabular}



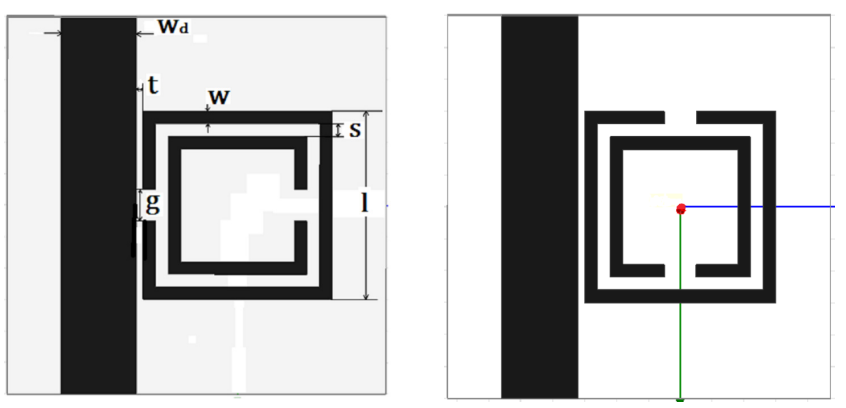

Figure 1. Layout of microstrip line loaded with Split Ring Resonator (a) SRR with gap parallel to the line (b) SRR with the gap perpendicular the line.
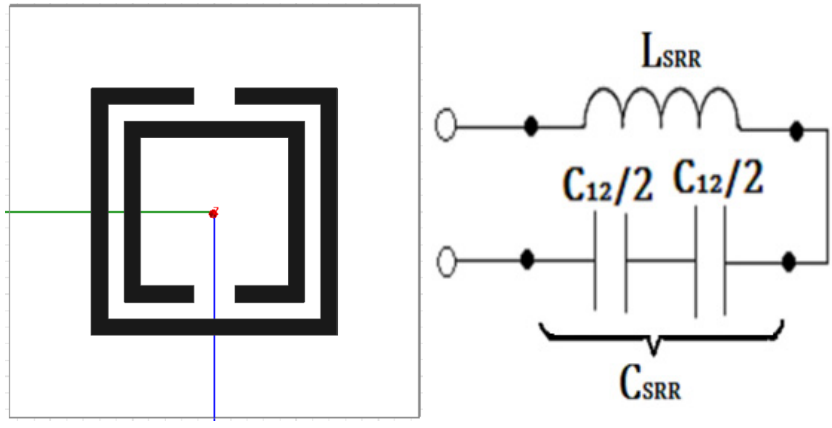

Figure 2. Split Ring Resonator (a) with two rings (b) Equivalent circuit of SRR with distribution capacitances

dimensions of SRR coupled to rectangular microstrip line are given in Table 1 and top view of SRR is shown in Figure 2(a).

In Figure 1(a), transmission line is $1.2 \mathrm{~mm}$ wider and loaded with SRR at $0.1 \mathrm{~mm}$ spacing. The length of the SRR is $3 \mathrm{~mm}$ and the width is taken as $0.2 \mathrm{~mm}$. The gap of split in both rings is $0.5 \mathrm{~mm}$ and spacing between the rings is $0.2 \mathrm{~mm}$. SRR is equivalent to LC circuit which is depicted in Figure 2(b). Total inductance $\left(\mathrm{L}_{\mathrm{SRR}}\right)$ is the length of SRR for two numbers of rings. The gap of SRR acts like capacitance. The distribution capacitance $\left(\mathrm{C}_{12} / 2\right)$ is the capacitance between the inner ring and outer ring.

The total inductance $\left(\mathrm{L}_{\mathrm{SRR}}\right)$ and total capacitance $\left(\mathrm{C}_{\mathrm{SRR}}\right)$ for SRR ring can be evaluated by using following equations:

The total inductance is expressed as:

$$
L_{S R R}=\frac{\mu_{0}}{2} \frac{l_{\text {avg }}}{\mathbf{4}} 4.86\left[\ln \left(\frac{0.986}{\rho}\right)+1.84 \rho\right]
$$

where, $\rho$ is the fill ratiowhich describes the hollowness of MSRR and is expressed for two number of rings

$$
\rho=\frac{(w+s)}{l-(w+s)}
$$

And $l_{\text {avg }}$ is the average strip length calculated as

$$
l_{\text {avg }}=4[l-(w+s)]
$$

Total capacitanceis expressed as

$$
C_{S R R}=\frac{1}{2}[2 l-3(w+s)] C_{0}
$$

where, $\mathrm{C}_{0}$ is per-unit-length capacitance between two parallel strips having width (w) and separation (s), given by

$$
C_{0}=\varepsilon_{0} \frac{K\left(\sqrt{1-k^{2}}\right)}{K(k)}
$$

$\mathrm{K}(\mathrm{o})$ is the complete elliptic integral of the first kind as used in $\stackrel{10}{ }$ and $\mathrm{k}$ can be expressed as

$$
k=\frac{\frac{s}{2}}{w+\frac{s}{2}}
$$

Subsequently the resonant frequency of SRR can be evaluated:

$$
f_{r}=\frac{1}{2 \pi \sqrt{L_{S R R} C_{S R R}}}
$$
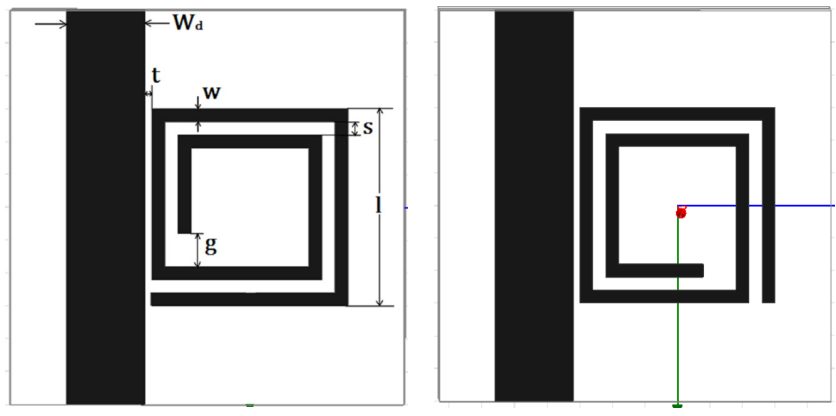

Figure 3. Layout of microstrip line loaded with Spiral Resonator (a) SR with gap parallel to the line (b) SR with the gap perpendicular the line.
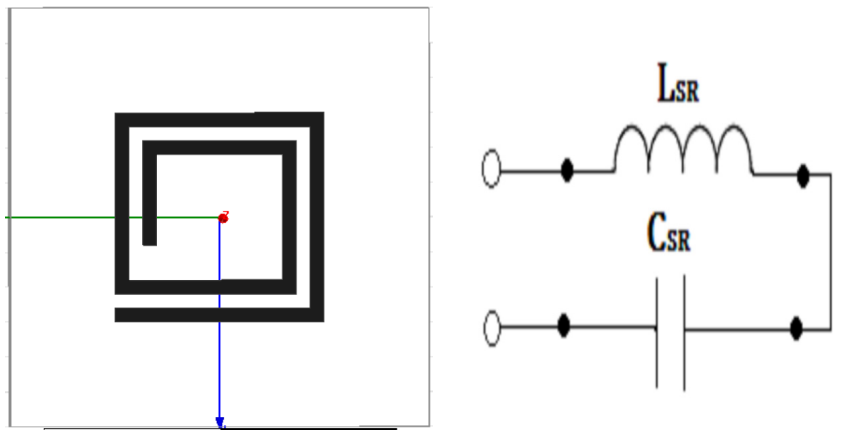

Figure 4. Spiral Resonator (a) Top view with two turns (b) Equivalent circuit of model. 


\subsection{SR Loaded Microstrip Line with Gap Parallel/Perpendicular to The Line}

We loaded microstrip line with SR in the same plane with gap parallel to the line as shown in Figure 3(a) and gap perpendicular to the line as presented in Figure 3(b).

In both the configuration, two turns of SR have been considered with spacing between two turns as 's'. Figure 4(a) and 4(b) shows the top view and equivalent circuit of the SR respectively.

Total inductance and capacitance of the SR can be calculated from the following equations:

The total capacitance $\mathrm{C}_{\mathrm{SR}}$ of dual turn $\mathrm{SR}$ is given by

$$
C_{S R}=4 C_{0}[l-1.5(w+s)]
$$

Total inductance for dual turn SR is given by

$$
L_{S R}=\frac{\mu_{0}}{2 \pi} l_{a v g}^{S R}\left[\frac{1}{2}+\ln \left(\frac{l_{a v g}^{S R}}{2 w}\right)\right]
$$

where, $\mu_{0}$ is free space permeability and is equal to $4 \pi \times 10^{-}$ ${ }^{10} \mathrm{~mm}$

The average total length and expressed for dual turn $\mathrm{SR}$ as

$$
l_{a v g}^{S R}=\frac{\mathbf{8} l-9(w+s)}{2}
$$

Hence the resonant frequency of SR can be evaluated as

$$
f_{r}=\frac{1}{2 \pi \sqrt{L_{S R} C_{S R}}}
$$

\section{Results and Discussion}

SRR/SR loaded microstrip line is numerically explored inside a waveguide with Ansoft software 'High Frequency Structure Simulator (HFSS)' to get the resonating frequency region. The Perfect Electric Conductor (PEC)

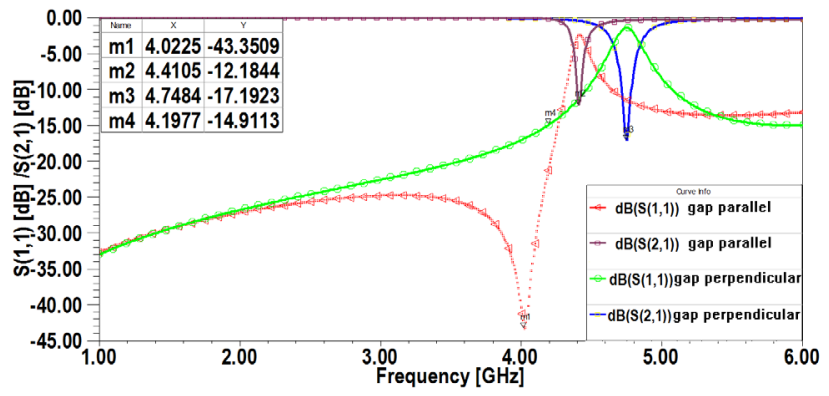

Figure 5. Reflection coefficient $\left(\mathrm{S}_{11}\right)$ and Transmission coefficient $\left(\mathrm{S}_{21}\right)$ of SRR loaded microstrip line with the gap parallel and perpendicular to the line. boundary conditions and the Perfect Magnetic Conductor (PMC) boundary conditions are applied on the appropriate faces of the unit cell. The two wave ports 1 and 2 are assigned to the rest of the sides of unit cell so as to excite negative permeability characteristics.

The reflection coefficient $\left(\mathrm{S}_{11}\right)$ and transmission coefficient $\left(\mathrm{S}_{21}\right)$ of SRR loaded microstrip line with the gap parallel and perpendicular to the line in Figure 5. It shows that resonant frequencies shift to higher frequency region as the gap of SRR changes from parallel to perpendicular and increase the return loss also. This is due to the reason that when gap of SRR is perpendicular to the line, an extra mutual capacitance is added which leads to increase in resonant frequency.

The reflection coefficient and transmission coefficient of isolated SRR analyzed in waveguide is shown in the Figure6. The isolated microstrip line resonates at 0.5 GHz. The resonant frequency of isolated microstrip line is much less than SRR loaded microstrip line. This is because in the SRR loaded microstrip line, a distribution capacitance is mutually induced with the strip line which results in increase in resonant frequency. It is seen that the simulated resonant frequency of SRR, $\left(\mathrm{f}_{\mathrm{rs}(\mathrm{SRR})}\right)$ is 3.8

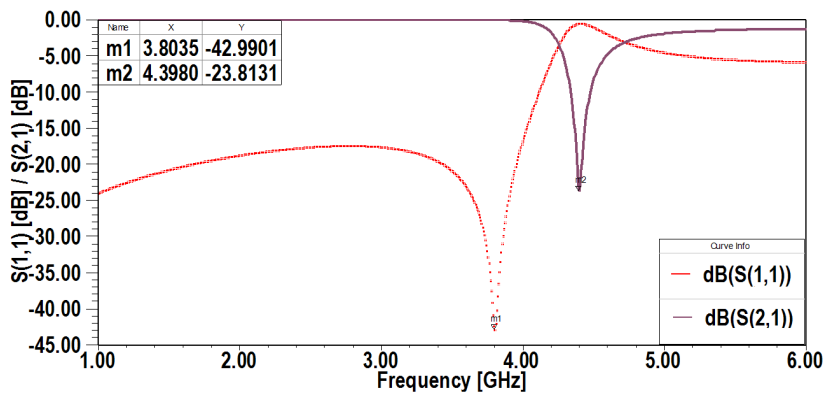

Figure 6. Reflection coefficient $\left(\mathrm{S}_{11}\right)$ and Transmission coefficient $\left(S_{21}\right)$ of SRR for two rings.

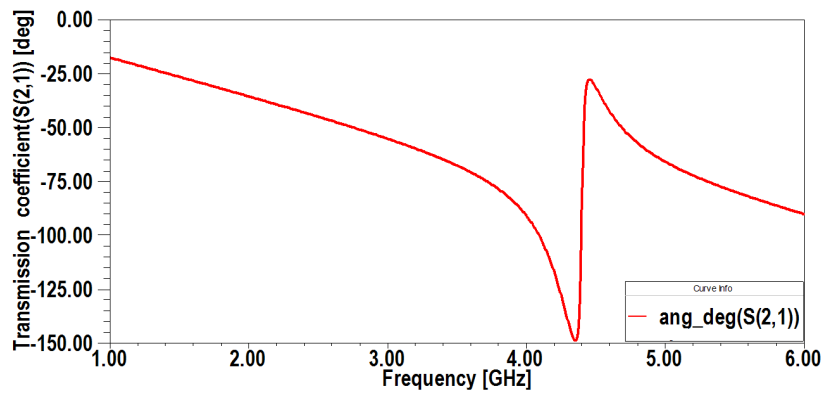

Figure 7. Reflection coefficient $\left(\mathrm{S}_{11}\right)$ and Transmission coefficient $\left(\mathrm{S}_{21}\right)$ of Spiral loaded to the microstrip line with the gap parallel and perpendicular to the line. 
$\mathrm{GHz}$ when it is analyzed in a rectangular waveguide without microstrip line. The calculated resonant frequency of SRR, $\left(\mathrm{f}_{\mathrm{rc}(\mathrm{SRR})}\right)$ from equation (7) is $3.5 \mathrm{GHz}$.

Figure 7 gives reflection coefficient and transmission coefficient of SR loaded microstrip line with the gap parallel and perpendicular to the line. The resonant frequency of SR loaded microstrip line with the gap perpendicular to the line is higher than the gap parallel to the line. It also illustrates that the resonant frequency of SR is much lower than that of SRR with same dimensional parameters.

It is observed from Figures 5 and 7, SR loaded microstrip line resonates at a frequency much less as compared to SRR loaded microstrip line with gap parallel and perpendicular to the line. The simulating resonant frequency increases appreciably with the same length of SRR without reduction of return loss. It is observed that SR resonates at same frequency as the SRR with scaled down dimensions.

Reflection coefficient and transmission coefficient of isolated SR is shown in Figure 8. The simulated resonating frequency of $\mathrm{SR}\left(\mathrm{f}_{\mathrm{rs}(\mathrm{SR})}\right)$ is $2.4 \mathrm{GHz}$ when examined in a rectangular waveguide without microstrip line, whereas calculated resonant frequency, $\left(f_{\mathrm{rc}(\mathrm{SR})}\right)$ from equation (11) is $1.8 \mathrm{GHz}$. A comparison of calculated

Table2. Calculated and simulated resonant frequency of SRR and SR

\begin{tabular}{|c|c|c|c|}
\hline Sr No. & $\begin{array}{c}\text { Type of } \\
\text { reonator }\end{array}$ & $\begin{array}{c}\text { Calculated value } \\
\text { of resonant } \\
\text { frequency }\left(\boldsymbol{f}_{\boldsymbol{r}}\right)\end{array}$ & $\begin{array}{c}\text { Simulated value } \\
\text { of resonant } \\
\text { frequency }\left(\boldsymbol{f}_{\boldsymbol{r}}\right)\end{array}$ \\
\hline 1 & $\begin{array}{c}\text { Split Ring } \\
\text { Resonator (SRR) }\end{array}$ & $3.5 \mathrm{GHz}$ & $3.8 \mathrm{GHz}$ \\
\hline 2 & $\begin{array}{c}\text { Spiral Resonator } \\
\text { (SR) }\end{array}$ & $1.7 \mathrm{GHz}$ & $2.3 \mathrm{GHz}$ \\
\hline
\end{tabular}

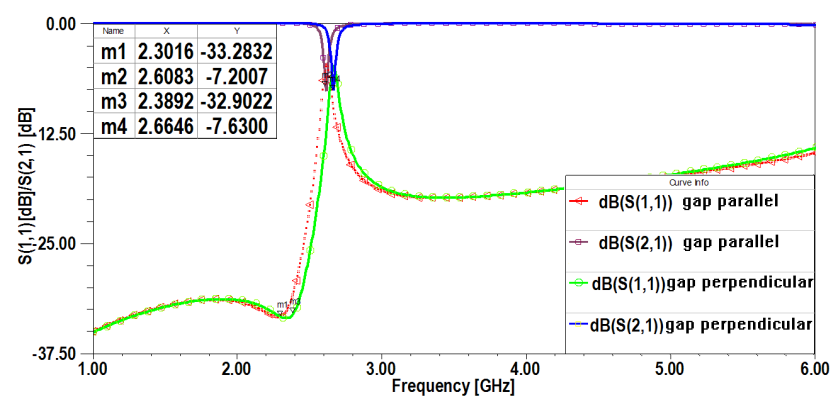

Figure 8. Reflection coefficient $\left(\mathrm{S}_{11}\right)$ and Transmission coefficient $\left(\mathrm{S}_{21}\right)$ of SR with two turns.

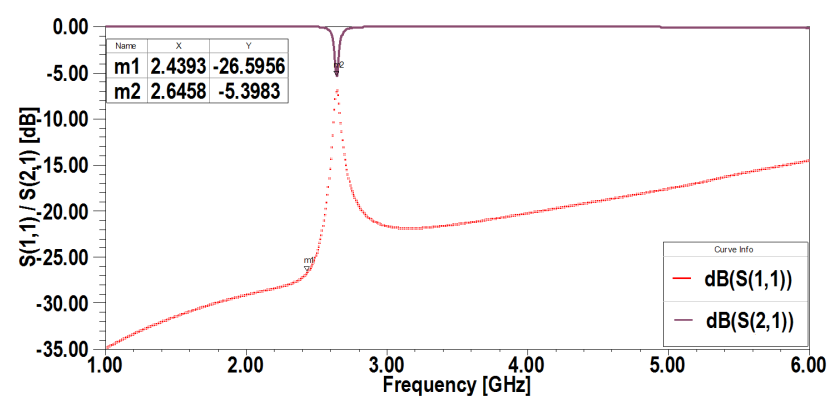

Figure 9. Phase of transmission coefficient $\left(\mathrm{S}_{21}\right)$ of $\mathrm{SRR}$ for two rings.

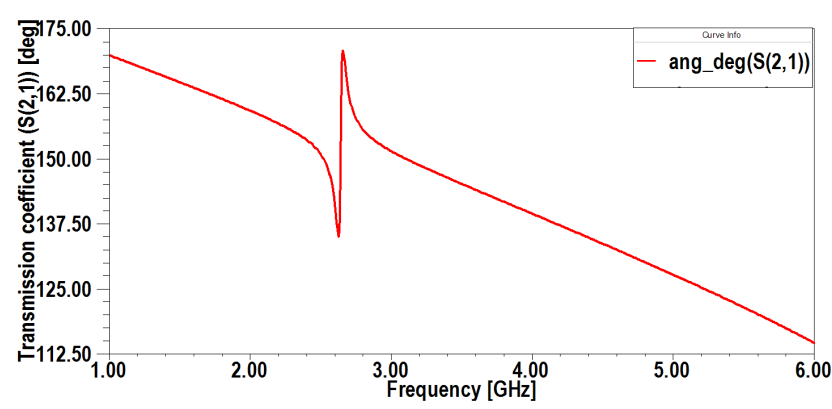

Figure 10. Phase of transmission coefficient $\left(\mathrm{S}_{21}\right)$ of SR for two turns.

and simulated resonant frequency of SRR and SR is tabulated in Table 2.

It is apparent from the table that the calculated and simulated resonant frequency is in close agreement with each other for both the resonators.

The phase of SRR with two rings and SR with two turns is shown in Figures 9 and 10 respectively. It is clear from the figures that the phase of transmission coefficient is reversed at the resonant frequency which is a feature of metamaterials. This validates the metamaterial behavior of SRR and SR.

\section{Conclusion}

In this paper, the magnetic resonance of SRR and SR loaded microstrip lines is presented with gap parallel and perpendicular to strip line. The simulated results of SRR loaded line and SR loaded transmission line are numerically evaluated with through the electromagnetic solver. It is evident from the results that the resonant frequency shifts to higher region as gap of SRR changes from parallel to perpendicular to the line with same dimension parameters. The same sized SR exhibits a high reduction in resonant frequency without affecting the return loss much than SRR. So, it can be surmised that the same sized 
SR resonate at comparatively lower resonating frequency as compared to SRR. Hence it is proposed that spiral resonator loading can be preferred to design an electrically small antenna by miniaturizing the dimensions to high scale than conventional SRR.

\section{References}

1. Eleftheriades GV, Balmain KG. Negative-refraction metamaterials: Fundamental principles and applications. New York: Wiley;2005.

2. Munk BA.Metamaterials: Critique and alternatives. New York: Wiley; 2009.

3. Veselago VG. The electrodynamics of substances with simultaneously negative values of $\varepsilon$ and $\mu$. Soviet Physics Uspekhi. 1968; 10(4):509.

4. Pendry JB, Holden AJ, Robbins DJ, Stewart WJ.Magnetism from conductors and enhanced nonlinear phenomena. IEEETransmission Microwave Theory Technology. 1999; 47(11):2075-84.

5. Shelby RA, Smith DR, Schultz S.Experimental verification of a negative index of refraction. Science Magazine.2001; 292(5514):77-9.

6. SmithDR, PadillaWJ, VierDC, Nemat-Nasser SC, SchultzS. Composite medium with simultaneously negative permeability and permittivity. Physical Review Letters.2000; 84(18):4184-7.

7. Engheta N, Richard WZ.Metamaterials: Physics and engineering explorations. Wiley \& Sons; 2006.

8. MarquésR, MesaF, MartelJ, MedinaF. Comparative analysis of edge- and broadside-coupled split ring resonators for metamaterial design theory and experiments. IEEE Transaction on Antennas and Propagation. 2003;51(10):2572-81.

9. BilottiF, ToscanoA, VegniL.Design of spiral and multiple split-ring resonators for the realization of miniaturized metamaterial samples. IEEE Transactions On Antennas and Propagation. 2007; 55(8):2258-67.
10. Rajni, Anupma M. Magnetic resonance of spiral resonators. International Journal of Applied Engineering Research. 2015; 10(13):33291-5.

11. Rajni, Anupma M. Resonance characteristics and effective parameters of new left hand metamaterial. Telkomnika Indonesian Journal of Electrical Engineering. 2015; 15(3):407-503.

12. GargTK, GuptaSC,PattnaikSS. Metamaterial loaded frequency tunable electrically small planar patch antenna. Indian Journal of Science and Technology. 2014; 7(11):1738-43.

13. Rajaraman G, AnithaM, MukerjeeA, SoodK, JyotiR. Dualband, miniaturized, enhanced-gain patch antennas using differentially-loaded metastructures.Indian Journal of Science and Technology. 2015; 8(1):11-16.

14. BaenaJD, BonacheJ, MartínF, SilleroRM, LasoMAG, García J. Equivalent-circuit models for split-ring resonators and complementary split-ring resonators coupled to planar transmission lines. IEEE Transactions on Microwave Theory and Techniques. 2005; 53(4):1451-61.

15. BojanicR, JokanovicB, MilosevicV. Reconfigurable delay lines with split-ring resonators. Microwave Review. 2011; 11(2):7-12.

16. Sindreu MD, NaquiJ, ParedesF, BonacheJ, Martín F. Electrically small resonators for planar metamaterial. Microwave Circuit and Antenna Design: A Comparative Analysis. Appl. Sci. 2012; 2(2):375-95.

17.Jordi N,Durán SM, MartínF. Modeling Split-Ring Resonator (SRR) and Complementary Split-Ring Resonator (CSRR) loaded transmission lines exhibiting cross-polarization effects. IEEE Antennas and Wireless Propagation Letters. 2013; 12:178-81.

18. BojanicR, MilosevicV, JokanovicB,Mena FM, Francisco M. Enhanced modelling of Split-Ring Resonators couplings in printed circuits. IEEE Transactions on Microwave Theory and Techniques. 2014; 62(8):1605-15.

19. Rajni, Singh G, Marwaha A. Modeling of Split Ring Resonators loadedmicrostrip line with different orientations. International Journal of Electrical and Computer Engineering. 2015; 5(6):1363-71. 